\section{SP0164 BARRIERS AND FACILITATORS TO ADHERENCE TO MEDICATION IN PATIENTS WITH INFLAMMATORY ARTHRITIS}

K. Koutsogianni. The Arthritis Foundation of Crete, Heraklion, Crete, Greece

Background: Inflammatory arthritis can cause pain, stiffness, joint damage and even disability without proper and early guided treatment. The full benefits of several treatments recommended, can be achieved if patients strictly follow drug regimens. However, rates of adherence to prescribed medications in patients with inflammatory arthritis seem to be suboptimal.

Objectives: To identify the barriers and facilitators for adherence to medication on patient's perspective and to suggest ways to facilitate the patients with chronic conditions as the inflammatory arthritis are, to understand the value of compliance to treatment.

Methods: The problem of poor patient compliance was detected about 2,000 years ago when Hippocrates taught his students to pay particular attention to the analysis of the causes that led their patients to lie regarding compliance with medical instructions given to them. The adherence to medication of patients suffering from chronic diseases reaches only $50 \%$ (WHO-2003) while the rate of compliance to prescribed medicines for patients with rheumatoid arthritis tend to be quite low and range from $30-80 \%$.

Fear and insecurity about the side effects of the medicines, the doubt of efficacy of medications, the refusal to accept the IA diagnosis and the long term treatments, the desire to stop treatment to be free of the ongoing dependency on medication, the lack of competence by some physicians to involve the patient in the treatment procedure and the large amount of patients co-payment are the most significant barriers to patients adherence. A survey conducted among patients of the Arthritis Foundation of Crete aiming to assess the degree of their adherence to MTX showed that 1 in 4 patients reported missing at least one dose of MTX on their own initiative during the last 6 months without referring to their doctor and the main reason was due to side-effects.

Experiencing beneficial effects of the medications and being able to maintain autonomy and social participation, receiving clear and understandable information about the treatment options, having support from family and friends, having a good relationship with the treating physician and being involved in shared decision making process are the keys for leading the patients to better adherence.

Conclusion: A good patient-health professional relationship, knowledge about treatment options and appropriate amount of information provided by HCPs as well as the patients' acceptance of their health condition and understanding of the necessity of long term therapy appear to improve adherence to medication for patients with inflammatory arthritis.

Disclosure of Interest: None declared

DOI: 10.1136/annrheumdis-2017-eular.7133

\section{SP0165 CAN MUSCULOSKELETAL ULTRASOUND INFORM HEALTH BELIEFS AND PLAY A ROLE FOR MEDICATION ADHERENCE?}

\section{K. Kumar. University of Manchester, Manchester, United Kingdom}

Rheumatoid Arthritis (RA) is a condition with no cure and can cause disability ${ }^{1}$ RA affects nearly 1 in 100 adults. Early disease is characterised by pain and other features of inflammation, such as heat, swelling of joints, and loss of function. RA is associated with increased costs of co-morbid conditions (such as cardiovascular (CVD) associated with $\mathrm{RA}^{2}$.

Medication can ease symptoms and limit disease progression in $\mathrm{RA}^{3}$. Despite this, nonadherence to medication is common in $\mathrm{RA}^{4}$. In the UK, we found that showing patients their joint swelling via musculoskeletal ultrasound scan as an educational tool helped to increase beliefs about the necessity of treatment ${ }^{5}$. Our study provided a deeper understanding of adherence to disease modifying anti-rheumatic drugs (DMARDs) in patients with RA. Improving adherence is likely to be facilitated by incorporating visual representations of the disease process and better explanations of the consequences of poorly controlled RA into the consultation. This session will uncover some suggestions around the use of musculoskeletal ultrasound in improving adherence to DMARDs.

\section{References:}

[1] Emery P. Evidence supporting the benefit of early intervention in rheumatoid arthritis. J Rheumatol, 2002;29 (2):3-8.

[2] John H, Kitas G, Toms T, Goodson N. Cardiovascular co-morbidity in early rheumatoid arthritis. Best Pract Res Clin Rheumatol 2009; 23(1):71-82.

[3] Smolen JS, Landewe R, Breedveld FC, Dougados M, Emery P, Gaujoux-Viala $C$ et al. EULAR recommendations for the management of rheumatoid arthritis with synthetic and biological disease-modifying antirheumatic drugs. Ann Rheum Dis 2010; 69(6):964-975.

[4] Pasma A, Schenk CV, Timman R, Busschbach J, van den bemt B, Molenaar E et al. Non-adherence to disease-modifying antirheumatic drugs is associated with higher disease activity in early arthritis patients in the first year of the disease. Arthritis Research \& Therapy 2015; 17:doi:10.1186/s13075-0150801-4.

[5] Kumar K, Raza K, Gill P, Greenfield S. The impact of using musculoskeletal ultrasound imaging and other influencing factors on medication adherence in patients with rheumatoid arthritis: a qualitative study. Patient Prefer Adherence 2016; 10:1091-1100.

Disclosure of Interest: None declared

DOI: 10.1136/annrheumdis-2017-eular.7085

\section{SATURDAY, 17 JUNE 2017 Outcome in juvenile idiopathic arthritis}

\section{SP0166 LONG-TERM OUTCOME OF JIA INTO ADULTHOOD}

B. Flato ${ }^{1,2}$. ${ }^{1}$ Institute of clinical medicine, University of Oslo; ${ }^{2}$ Dep of Rheumatology, Oslo University Hospital, Oslo, Norway

Background: In patients with juvenile idiopathic arthritis (JIA), scarce information on disease outcomes into adulthood are available.

Objectives: To review the long-term outcome and possible time trends in adult JIA and discuss how JIA in adulthood is similar to or different from adult onset rheumatic diseases.

Results: JIA is a heterogeneous group of diseases, but across different categories patients with JIA share some clinical, prognostic and genetic characteristics different from those found in adult onset arthritis (i.e. growth disturbances, more varying prognoses, and HLA-DRB1*08). The most common categories of JIA, oligoarthritis and RF-negative polyarthritis, do not have any adult counterpart. On the other hand, $82-96 \%$ of patients with systemic, RF-positive polyarticular, psoriasis related, and enthesitis related JIA were recently found to fulfil the criteria for adult Stills, RA, psoriatic arthritis, and adult spondylarthropathy, respectively. Furhermore, recent analyses of large patient groups show that patients with oligoarticular and RF-negative polyarticular JIA share HLA associations with seronegative RA (HLA-DRB1 amino acid at position 13).

At transition to adult care, about half of the JIA patients (range 42-67\%) have active disease or are on anti-rheumatic medication. In adult JIA patients, active disease after more than 20 years of disease duration occured in 40 percent (range $37-43 \%$ ). Differences in definitions of remission, classification criteria and/or selection of patient may explain these variations.

Within a cohort, no reduction were found in the proportion of patients with active JIA from childhood to adulthood in 3 long-term longitudinal studies; $62 \%$ at 5 - and $60 \%$ at 17 -year followup, $42 \%$ at 10 - and $37 \%$ at 26 -year followup) and $45 \%$ at $15-$ and $41 \%$ at 30 -year followup.

Limitation in physical functioning has been found in $45-50 \%$ of adult JIA patients The proportion with severe disability has increased with increased disease duration in several cross sectional and one longitudinal study. However, no definite trends towards detoriation of physical functioning over time were found in two recent longitudinal studies. The proportion of JIA patients with severe disability was low in long-term studies from this century, $3 \%$ to $11 \%$, compared to $15-48 \%$ in studies from 1960 to 1990.

Up to $90 \%$ of the patients with childhood polyarthritis had radiographic damage in studies from 1966-76, compared to about $40 \%$ in studies from this century. In the total group of JIA patients, $24 \%>36 \%$ developed erosive disease, most frequently in wrists and hips. However, the use of radiographic scoring systems in JIA has been hindered by growth disturbances and varying joint distributions. Thus radiographic progression has never been investigated in clinical trials.

Several studies have shown that adults with JIA experience more pain, poorer health related quality of life and lower employment rates than age and sex matched controls from the general population, in spite of similar or higher education levels. A new study shows that JIA has a detrimental effect on HRQOL both in patients with and without clinical remission, and physical, but not mental, HRQOL deteriorate slightly from adolescence to adult life.

Ongoing active uveitis into adulthood has been found in half of the patients with JIA associated uveitis. One third of them have visual impairment and $40 \%$ need ocular surgery in early adult life. Recent studies indicate that adult JIA patients have increased rates of subclinical atherosclerosis and malignancies.

The use of arthroplasties in JIA patients decreased dramatically from 1990 to 2005 , while the rates were stable in RA and increased in spondylarthropathy and osteoarthritis patients. Joint surgery now occurs in less than $15 \%$ of adult JIA patients.

Previous long-term followup studies have mainly been performed before biologic agents were available or early methotrexate was used. Long-term outcomes in JIA patients treated with biologic agents have been described in two registry based studies. Inactive disease was achieved in $24-67 \%$ of the adult JIA patients and $52-74 \%$ were still on a biologic agent at last followup. It has been found that biologics improve physical HRQOL in children with JIA, and that most of the acquired improvement is maintained in adult life. However, biologics were started several years after disease onset in these studies. Only $38-56 \%$ of adult JIA patients with long-term active disease were on synthetic or biological DMARDs according to recent followup studies.

Conclusion: About half of JIA patients have active disease into adulthood. JIA affects physical outcome, HRQOL and visual outcome, but adult patients with long-term active JIA seems to receive inadequate therapy. So far, the long-term outcome in patients receiving early biologic and/or synthetic DMARDs is not known.

Disclosure of Interest: None declared

DOI: 10.1136/annrheumdis-2017-eular.7138 